\title{
The Retrospect and the Prospect of a Research on Pronouns in Excavated Materials of the Warring States
}

\author{
Yanmei Zhang \\ College of Liberal Arts \\ South China Normal University \\ Guangzhou, China \\ School of Foreign Language \\ Guilin University of Electronic Technology \\ Guilin, China
}

\author{
Yujin Zhang \\ College of Liberal Arts \\ South China Normal University \\ Guangzhou, China
}

\author{
Han Ding \\ School of Foreign Language \\ Guilin University of Electronic Technology \\ Guilin, China
}

\begin{abstract}
Nowadays, the grammatical study in excavated materials has been attracting more attention of scholars. This article, based on the differences of the corpus of researchers, reviews the studies according to pronouns in Excavated Materials of the Warring States from four aspects: grammar and pronouns study of bamboo slips used in the Qin Dynasty, grammar and pronouns study of bamboo slips and silks used in the Chu Dynasty, grammar and pronouns study of bronze script of the Warring States as well as grammar and pronouns study of several excavated materials of the Warring States. It not only affirms the achievements but also points out shortages of the study. The shortages are that it lacks periodical monographic studies about Chinese pronouns in excavated materials of the Warring States; it rarely fully uses a variety of the corpora of excavated materials of the Warring States and the number of scholars concentrating on pronouns in excavated materials of the Warring States is not especially high, currently making the works of a fully operational comparative study on the correlation of synchronic and diachronic not yet. On this foundation, the article prospects the future study of pronouns in excavated materials of the Warring States.
\end{abstract}

Keywords-excavated Materials; the Warring States; pronouns

\section{INTRODUCTION}

Strengthen cultural heritage protection and implement the project of Chinese ancient books and records are important parts of constructing Chinese excellent traditional culture inheritance system. In recent decades, especially after 1980s, there have been constantly materials of the Warring States and the Qin Dynasty excavated in domestic China. Excavated materials, with a long period buried underground and without being spread, are able to really retain the appearance of the language at that time and possess valuable corpus value. The discovery of excavated materials definitely provides each course rich and broad resources especially in language study. It is the research on Chinese grammar by using unearthed documents and materials that is becoming a new field of the study of ancient Chinese grammar.

In the past, studies on pronouns in Excavated Materials of the Warring States were mostly using documents handed on from ancient times. Meanwhile, it rarely made synchronic contrast with pronouns in excavated materials and even lacked systematic comparison.

When it comes to studying the pronouns of materials of the Warring States, it should be accommodated that fully using the corpora of excavated materials of the Warring States, based on excavated materials of the Warring States as fundamental corpora, collecting every example of every pronoun of all excavated materials of the Warring States, analyzing one by one and categorizing so as to make static description and necessary statistics for every pronoun. Besides, it should be ensured the authenticity of the corpora, using historical materials of the Warring States as circumstantial evidences. When both of them have a thorough and detailed comparison, it is better to leave a deficiency uncovered than to have it covered without discretion. Moreover, when facing complex language phenomenon in the course of the study, it should be considered in many ways as much as possible and made cautious conclusions. This kind of study will be highly innovative and extremely valuable.

\section{THE RETROSPECT OF A RESEARCH ON PRONOUNS IN EXCAVATED MATERIALS OF THE WARRING STATES}

The study of Chinese pronouns has always been a concern of scholars. Thus, before Ma Shi Wen Ton was born, there were ancient Chinese scholars doing research on 
Chinese pronouns. After Ma Shi Wen Ton was written, plenty of scholars do research on Chinese pronouns. With regard to the study of ancient Chinese pronouns, papers using historical documents as corpora have always been a lot; in some monographs, they have also been discussed.

In recent years, the grammatical study in excavated materials has been attracting more attention of scholars and obtained many results. Papers relate to pronouns in excavated materials quite a lot. Many scholars, like Gao Benhan, Hu Shi, Rong Geng, Gao Mingkai, Sun Xiju, Zhou Fagao, Huang Shengzhang, Han Yaolong, Zhou Shengya, Guan Xiechu, Ma Guoquan, Tang Yuming, Zhao Shiju, Yang Bojun, Chen Weizhan, Qian Zongwu, Yao Zhenwu, Hong Bo and so on, have involved the issue of pronouns in excavated materials in their works more or less. There are also some monographs referring to pronouns in excavated materials. Those books mainly are The Oracle Inscriptions of Yin Ruins by Mr. Chen Mengjia, The Grammar Study of The Oracle-bone Inscriptions of Yin Ruins by Mr. Guan Xiechu, The Exploration of Function Words of Oracle by Mr. Zhao Cheng, Inscriptions Grammar of Yin Ruins by Mr. Li $\mathrm{Xi}$, Oracle Grammar, The study of Chinese grammar in West Zhou Dynasty, The study of Chinese pronoun in West Zhou Dynasty by Mr. Zhang Yujin. Further more, predecessors have done many related research achievements on the basis of the research on pronouns in excavated materials of the Warring States. According to different materials used by researchers, we will review the research from the following four aspects:

\section{A. Grammar and Pronouns Study of Bamboo Slips Used in the Qin Dynasty}

Firstly, the monograph The Grammar Study of Shuihudi Qin Tomb Bamboo Slips(Wei Desheng, 2000) is a book comprehensively describing the grammatical problems with Shuihudi Qin tomb bamboo slips as the study subject. Chapter 2 of the book is number and addressing, studying numerals, quantifiers and pronouns of Shuihudi Qin tomb bamboo slips. Secondly, there are also some monographic study results. For instance, The Copula "Shi" in Shuihudi Qin Tomb Bamboo Slips (Shi Feng, 2000) exhaustively coordinated "Shi" in Shuihudi Qin Tomb Bamboo Slips, considering that "Shi" in Shuihudi Qin Tomb Bamboo Slips has the usage of demonstrative pronoun, structural particle and copula. Another example is that Some Researches on Grammatical Phenomenon of Shuihudi Qin Tomb Bamboo Slips (Feng Chuntian, 1984) discussed demonstrative pronouns "shi" and "ci" in Shuihudi Qin Tomb Bamboo Slips. Besides, The Pronoun Research on Bamboo Slips Used in the Qin Dynasty written by $\mathrm{Hu}$ Weizeng, systematically and comprehensively studied first, second and third personal pronouns in bamboo slips used in the Qin Dynasty. Apart from Shuihudi Qin tomb bamboo slips, the corpora also have Longgang Qin bamboo slips and Guan Ju Qin and Han bamboo slips.

\section{B. Grammar and Pronouns Study of Bamboo Slips and Silks Used in the Chu Dynasty}

Initially, Grammar Study of Bamboo Slips in the Chu Dynasty of the Warring States (Li Mingxiao, 2010) is the first book carrying out a comparatively comprehensive study on the grammar study issue of Bamboo Slips in the Chu Dynasty. The book, using quite a lot of newly excavated materials, is divided into two parts, morphology and syntax. Among seven chapters of morphology, pronoun, chapter one, studying personal pronouns, demonstrative pronouns and interrogative pronouns of bamboo slips in the Chu Dynasty of the Warring States. Then, the results of thematic studies like The Vocabulary Study of Baoshan Chu Slips (Wang Ying, 2004). The whole book contains eleven chapters, pronoun, chapter six, analyzing the meaning and usage of nine pronouns of Baoshan Chu Slips. Additionally, it is Grammar Reading Notes of Shanghai Museum Bamboo Slips (One or Two Letters) (Zhou Shoujin, 2006) that discusses the grammatical features of bamboo slips in Shanghai Museum. It includes functionary differentiations of the pronouns "shi", "ci" as well as "A, is also B" and "A, that is B".

\section{Grammar and Pronouns Study of Bronze Script of the Warring States}

Grammar research books totally using bronze script of the Warring States as corpora are not many. Mainly, books referring to pronoun research are Philological Study of Zhongshan Wang Bronze Inscription (Jiang Yunyu, 2001) and discussing a Special Use of "Wo" of Bronze Script in Various States (Daxi Keye, 1998). The first book mainly takes Zhongshan Wang Tenmi, Zhongshan Wang Square Pot Inscription and Pot Inscription of the Warring States as study objects, in which chapter five concentrates on the grammatical phenomena reflected by inscriptions and talks about the synonymy generalization of "jue" and "qi" in Zhongshan Wang Bronze Inscription. The other book studies a special use of "Wo" of Bronze Script in Various States.

\section{Grammar and Pronouns Study of Several Excavated Materials of the Warring States}

Excavated materials of the Warring States can be divided into bamboo slips used in the Qin Dynasty, bamboo slips and silks used in the Chu Dynasty, bronze script of the Warring States and jade text of the Warring States. Several excavated materials of the Warring States refer to including at least two materials mentioned above. The results of utilizing several excavated materials of the Warring States to do comprehensive grammar researches currently mainly include a Research on Function words in Excavated Materials of the Warring States (Zhang Yujin, 2011). This book studies four excavated materials of the Warring States that are bronze script of the Warring States, scripts from bamboo slips of the Warring States, books copied on silk of the Warring States and jade text of the Warring States. It covers the discussions that whether "zhe" and "suo" are pronouns and that relates to the blur of pronoun and multiple categories of pronoun and function word. In addition, books, utilizing several excavated materials of the Warring States to do monographic study, 
referring to pronouns are Grammar Research on Excavated Materials of the Warring States (Zhou Shoujin, 2005). The whole book, using bamboo slips used in the Qin Dynasty, bamboo slips used in the Chu Dynasty and bronze script of the Warring States, is divided into four chapters. Chapter three is conjoined element, mainly studying three groups of words, in which "shi" and "ci" is part of it.

\section{THE PROSPECT OF A RESEARCH ON PRONOUNS IN EXCAVATED MATERIALS OF THE WARRING STATES}

From section two mentioned above, we have made the retrospect of grammars in excavated materials of the Warring States especially pronouns. It can be seen that predecessors have made many achievements in this research field and it lays a good foundation for further study. But we should also see that former researches have limitations. Firstly, in Chinese academia, it has not been seen books comprehensively study grammar of Chinese pronoun system of the Warring States and periodical monographic studies about Chinese pronouns in excavated materials of the Warring States. In addition, researches, relating to pronouns in excavated materials of the Warring States, from using corpus, the more use a particular corpus, the less comprehensively use excavated materials of the Warring States. Thirdly, from the research team, although more and more scholars has been paying attention to the research on grammar and pronoun in excavated materials of the Warring States, not many of them devote themselves to this aspect. At present, it temporarily lacks not only periodical monographic studies about Chinese pronouns in excavated materials of the Warring States but also comprehensively and systematically make horizontal synchronic comparison with pronoun materials of the Warring States handed down from ancient times. Moreover, it lacks pronoun books that comprehensively and systematically make longitudinal diachronic comparison with pronoun materials of the Warring States handed down from ancient times with the Shang oracle, West-Zhou inscriptions on bronzes and any other period. Thus, it should be said that researches of this field still need to strengthen.

Recently, in domestic China, corpora of excavated materials of the Warring States that have been announced mainly are bronze script of the Warring States, scripts from bamboo slips of the Warring States (including Xinyang bamboo slips of Chu, Wulipai bamboo slips of Chu, Angtian Lake bamboo slips of Chu, Yang Jia Wan bamboo slips of Chu, Wang Mountain bamboo slips of Chu, Guo Dian bamboo slips of Chu, Shanghai Museum bamboo slips of Chu, Xincai bamboo slips of Chu, bamboo slips in Chinese University of Hong Kong, Shuihudi bamboo slips of Qin, Shuihudi bamboo tablets of Qin, Qingchuan bamboo tablets of Qin, Fangma Beach bamboo slips of Qin, Yue Shan bamboo tablets of Qin, Longgang bamboo slips of Qin, Choujiatai bamboo slips of Qin, Liyebamboo slips of Qin and Tomb of Marquis Yi of Zeng bamboo slips), book copied on silk of the Warring States (like book copied on silk of the Warring States in Changsha bullet Library) and jade text of the Warring States (including Yin jade marginal inscription of Qin, Xing Qi jade inscription, jade Huang admonitions carved on a stone, Shou Qiu lithoglyph, ten Qin kingdom inscribed steles and the engravings on Mount Yi).

Meanwhile, with thousands of years, China also has many valuable material corpora of the Warring States handed down from ancient times. These corpora mainly are Zuozhuan, Guoyu, The Analects of Confucius, Mozi, Lao Zi, Strategies of the Warring States, Chuang-tzu, Han Feizi, The Words of Mencius, The book of Xunzi, Lu Shi Chun Qiu. Materials handed down from ancient times, through a long period of spreading, repeatedly copying and collating, are inevitably distorting. As corpora, they have great limitations. However, looking from the language features inheritance of materials handed down from ancient times; many of them are relatively reliable and could provide good circumstantial evidence to the pronoun research of excavated materials of the Warring States.

According to the research on pronouns in excavated materials of the Warring States, we can consider to fully using a variety of the corpora of excavated materials of the Warring States, fully use a variety of the corpora of materials of the Warring States handed down from ancient times and make synchronic comparison between two corpora. Through theoretical approach, literature analysis, the combination of quantitative analysis and qualitative analysis and the combination of synchronic and diachronic studies, the true face of the pronoun system of the Warring States reflected by excavated materials and materials of the Warring States handed down from ancient times can be more totally systematically demonstrated. Besides, in the study of pronouns in excavated materials of the Warring States, we can put pronoun in excavated materials of the Warring States into the long river of Chinese historical development furtherly. Also, it is a way to compare it with the pronoun excavated from materials of Shang, West Zhou Dynasty, the Spring and Autumn Period handed down from ancient times and the pronoun in materials of Qin to early Han Dynasty, the Western Han Dynasty and the period after the Western Han Dynasty to make essential longitudinal diachronic comparison. Moreover, investigate its origin and development dynamically and reveal the origin and development of the reason and the law -- this kind of diachronic comparison will have a good theoretical reference value when establishing a reliable development history of Chinese pronoun and even a reliable development history of Chinese grammar.

What's more, although predecessors have achieved fruitful results by doing massive researches of Chinese pronouns of ancient times, they also need consultations. According to the research on pronouns in excavated materials of the Warring States, when studying, we can base on the research on pronouns in excavated materials of the Warring States and make deeper discussion to some issues that Chinese pronoun of ancient times in the current academic circle - make proof or disproof to the conclusion already existing and put forward insights and ideas on the basis of predecessors' researches. 


\section{CONCLUSION}

It can make sense theoretically that complying with the new trend of the study of ancient Chinese grammar, fully using several excavated material corpora of the Warring States, do research to excavated material of the Warring States from microscopic to macroscopic and comprehensively and systematically study. Besides, making comprehensively and systematically synchronic comparative study to excavated material of the Warring States and material pronoun of the Warring States handed down from ancient times, comparing the similarities and differences and analyzing the reason, discuss the pronoun system of the Warring States showing from excavated materials and materials handed down from the ancient times. Meanwhile, it is a wise method to put pronoun in excavated materials of the Warring States into the long river of Chinese historical development, comparing it with the excavated materials and the materials handed down from ancient times of the Shang oracle, West Zhou bronze inscriptions to make essential longitudinal diachronic comparison, investigating its origin and development dynamically and revealing the origin and development of the reason and the law. Specific performance in:

- Initially, making comprehensively and systematically synchronic comparative study to excavated material and material pronoun of the Warring States handed down from ancient times, compare the similarities and differences and analyzing the reason; discuss the pronoun system of the Warring States showing from excavated materials and materials handed down from the ancient times. Some new corpora, fresh research methods, new reliable conclusions and fresh evidences of the process of synchronic comparison have significant role of theoretical support when digging the concrete facts of Chinese and enriching research theory and method of Chinese pronoun.

- Secondly, according to the different times of formation, excavated materials of the Warring States can be divided into early and late two stages, investigating the pronoun evolution from early to late and discussing the law and reason of changes. Additionally, the pronoun system of excavated materials of the Warring States can be used as a coordinate, which makes a diachronic comparison with the pronoun of excavated materials and the pronoun handed down to Shang, West Zhou and the Spring and Autumn Period so as to touch its direct source. Also, it is worth comparing it with the pronoun in materials of Qin to early Han Dynasty, the Western Han Dynasty and the period after the Western Han Dynasty to make essential longitudinal diachronic comparison and investigating its changes to discuss the reason and law of evolution. This kind of diachronic comparison has good theoretical reference value to establishing a reliable Chinese grammar history especially a reliable development history of Chinese pronoun.

\section{ACKNOWLEDGEMENT}

This research was financially supported by the Project of Undergraduate Teaching Reform of Higher Education in Guangxi in 2016, with the project index number 2016JGA198, in which the content was the reform and research of "practical teaching" of International Chinese Education Specialty on the base of universities personnel training in Guangxi.

\section{REFERENCES}

[1] Ma Jianzhong. Ma Shi Wen Ton [M]. Beijing: The Commercial Press, 1898.

[2] Chen Mengjia. The Review of Divination Inscriptions of Yin Ruins [M]. Beijing: The Science Publishing Company, 1956.

[3] Zhang Yujin. A Research on Chinese Pronoun of The Western Zhou Dynasty [M]. Beijing: Zhong Hua Book Company, 2006.

[4] Zhang Yujin. A research on Function words in Excavated Materials of the Warring States [M].Beijing: People press, 2011.

[5] Zhang Yujin. The Retrospect and the Prospect of A Research on Grammar in Excavated Materials of the Warring States [J]. Journal of South China Normal University (JCR Social Science Edition), 2007, 06: $55-62+159$ 